\title{
Uric acid lowering improves insulin sensitivity and lowers blood pressure: a meta-analysis of randomized parallel-controlled clinical trials
}

\author{
Qunchuan Zong\#, Guanyi Ma\#, Tao Wang
}

\begin{abstract}
Division of Orthopedic Surgery, the Affiliated Hospital of Qinghai University, 810001, Xining, Qinghai Province, China.
\end{abstract}

\#Q.Z. and G.M. contributed equally to this work.

\begin{abstract}
Objectives: This meta-analysis aimed to investigate whether uric acid lowering treatment can improve $\beta$-cell function and insulin sensitivity.

Methods: PubMed, Cochrane Library, EMBASE and China Biology Medicine were searched up to March 1, 2020. Randomized controlled clinical trials of urate lowering therapy in hyperuricemia patients were included in meta-analysis. Effect size was estimated as mean difference with $95 \%$ confidence interval (CI).

Results: Our search yielded 7 eligible trials with 503 participants. This meta-analysis showed that uric acid-lowering therapy decreased fasting insulin $-1.43 \mu \mathrm{IU} / \mathrm{ml}$ (weighted mean differences (WMD, 95\% CI -2.78 to -0.09), homeostasis model assessment of insulin resistance -0.65 (WMD, 95\% CI -1.05 to -0.24), systolic blood pressure $-2.45 \mathrm{~mm} \mathrm{Hg}$ (WMD, 95\%CI -4.57 to -0.33 ) and diastolic blood pressure $-3.41 \mathrm{~mm} \mathrm{Hg}$ (WMD, 95\%CI -3.87 to -2.95 ). However, the treatment had no significant effect on fasting plasma glucose (WMD $-0.19 \mathrm{mmol} / \mathrm{L}, 95 \% \mathrm{CI}-0.42$ to 0.05 ), homeostasis model assessment of $\beta$-cell function index (WMD $-0.02,95 \% \mathrm{CI}-0.28$ to 0.24 ), total cholesterol (WMD $0.18 \mathrm{mg} / \mathrm{dl}$; $95 \% \mathrm{CI},-1.39$ to 1.75 ) and triglyceride (WMD $3.15 \mathrm{mg} / \mathrm{dl}, 95 \%$ CI -9.83 to 16.14 ).

Conclusion: Uric acid-lowering therapies might improve insulin sensitivity and lower blood pressure, but had no significant effect on HOMA- $\beta$ and serum lipids.

Keywords: Hyperuricemia; uric acid lowering treatment; $\beta$-cell function; insulin sensitivity.

DOI: https://dx.doi.org/10.4314/ahs.v21i1.13

Cite as: Zong Q, Ma G, Wang T. Uric acid lowering improves insulin sensitivity and lowers blood pressure: a meta-analysis of randomized parallel-controlled clinical trials. Afri Health Sci. 2021;21(1):82-95. https:// dx.doi.org/ 10.4314/abs.v21i1.13
\end{abstract}

\section{Background}

Metabolic syndrome is a cluster physiological and anthropometric abnormalities of nutrients metabolism, such as hyperglycemia, hyperuricemia and hyperlipidemia $^{1,2}$. The metabolism of three major nutrients are closely linked. Hyperuricemia could contribute to abnormal glucose metabolism, insulin resistance (IR), even pancreatic $\beta$-cell death ${ }^{3-7}$. Substantial data from epidemiologic and experimental studies indicate an emerg-
Corresponding author:
Tao Wang,
Division of Orthopedic Surgery,
the Affiliated Hospital of Qinghai
University, 810001, Xining,
Qinghai Province, China
Tel / Fax: +860971 6230766
E-mail:wt17809711598@163.com

ing association between hypruricemia, type 2 diabetes mellitus (T2DM), and cardiovascular-related diseases. ${ }^{8}$ Several studies revealed that hyperuricemia may be an independent risk factor for the development of T2DM 9-13, which suggest a substantial implication for a correlation between uric acid concentration and insulin resistance (or insulin sensitivity) ${ }^{13-15}$. Also, hyperuricemia is substantially implicated in cardiovascular risks, ${ }^{16-18}$ vascular complications, ${ }^{19}$ the further long-term cardiovascular events $^{13}$ and mortality ${ }^{19}$ in T2DM patients. Pharmacologic agents lowering serum UA proved to play a promising role in the management of T2DM and CVD related disease ${ }^{8}$. Asymptomatic hyperuricemia is a common entity faced by physicians in day to day practice. Although there are clear recommendations on the treatment of gout with urate lowering therapy (ULT), the management of asymptomatic hyperuricemia remains controversial, especially in diabetic patients with 
asymptomatic hyperuricemia. Based on above observation, we conduct a meta-analysis to investigate whetherLT might be helpful for diabetes patients.

\section{Materials and methods \\ Design}

The meta-analysis followed the Preferred Reporting Items for Systematic Reviews and Meta-Analyses (PRISMA) guidelines for meta-analyses of interventional studies.

\section{Data sources and search strategy}

English and Chinese language publications were identified from MEDLINE, EMBASE, Cochrane Library and Chinese Biomedical Literature Database (CBM) from inception to May 2016. Search strategy was as follows: ("Hyperuricemia/drug therapy" [Mesh] AND "Diabetes Mellitus" [Mesh]) OR ((diabetic OR glucose) AND (uric acid)) AND (randomized controlled trial [pt] OR controlled clinical trial [pt] OR randomized [tiab] OR placebo [tiab] OR clinical trials as topic [mesh: noexp] OR randomly [tiab] OR trial [ti]) NOT (animals [mh] NOT humans [mh]).

\section{Study selection}

Eligible studies fell into four categories, including (1) randomized controlled trials (RCTs); (2) participates were hyperuricemia patients in adults with or without diabetes, and hyperuricemia is defined with the World Health Organization (WHO) diagnostic criteria; (3) intervene with hypouricemic agents except losartan and fenofibrate (because of their effect on blood pressure and insulin sensitivity ); (4) reported at least fasting plasma glucose (FPG), fasting insulin (FINS) and homeostasis model assessment of insulin resistance (HOMA-IR) outcome data.

Meanwhile, we excluded the studies that were cross-sectional studies, cohort studies or studies on animals; or that were duplicated. If the same population was reported in more than one studies, we included the one with the most complete data.

\section{Data extraction and synthesis}

For each eligible trial, we extracted data on name of the first author, year of publication, study design, participants' characteristics, sample size for each group, intervention period, contents of intervention and control conditions, as well as main study outcomes.

Outcome measures were classified into three aspects:
(1) $\beta$-cell function as reflected changes in FPG, FINS and homeostasis model assessment of $\beta$-cell function (HOMA- $\beta$ ); (2) the level of IR was reflected as change in HOMA-IR; (3) cardiovascular risk factors including changes in total cholesterol (TC), triglyceride (TG) and blood pressure (BP).

\section{Quality assessment}

The quality of the individual studies was assessed by the Cochrane Risk of Bias tool ${ }^{20}$. The categories were random sequence generation, allocation concealment, blinding (participants, study personnel and outcome assessment), incomplete outcome data addressed, selective outcome reporting and other sources of bias. Quality was rated as 'high' if at least the first three criteria (adequate sequence generation, allocation concealment and blinding) were fulfilled and not more than one of the others was rated 'unclear'. Quality was rated as 'low' if these first three or any other four criteria were rated as unclear or inadequate. All the otherwere rated as 'medium' quality. Two investigators independently evaluated the above risk of bias domains and consensus was achieved through a third reviewer.

The pooled analyses were performed using STATA version 12.0. The Cochrane Q and $\mathrm{I}^{2}$ statistics was carried out to assess heterogeneity across studies. For the Q statistic, a level of $\mathrm{P}$ value $\leq 0.10$ was considered statistically significant for heterogeneity; for $\mathrm{I}^{2}$, values of 25, 50 and $75 \%$ indicate low, moderate and high levels of heterogeneity, respectively ${ }^{21}$ 22. Weighted mean difference (WMD) and 95\% CIs between ULT group and the control were calculated as the effect size for continuous outcomes ${ }^{23}$. We performed primary analyses using a random effects model that adequately accounts for between-study variability. Sensitivity analyses and subgroup analyses were also performed when required. Furthermore, potential publication bias was assessed by Begg's ${ }^{24}$ funnel plot and Egger's ${ }^{25}$ regression test and a two-sided P-value $<0.05$ was considered statistically significant.

\section{Results}

\section{Search results}

A flowchart of the study selection process was presented in Figure 1. We conducted a literature search in PubMed, EMBASE and the Cochrane library from inception to October 25, 2019. We initially searched a total of 796 citations from the PubMed database, EMBASE, Cochrane Library and CBM, of which 615 cita- 
tions were excluded after the first screening based on titles and abstracts. They were excluded for the following main reasons: a total of 36 studies were duplicated and exposure or outcomes of 332 studies were not relevant. There were 227 studies reported as cross-sectional study, cohort study or other publication type. And 20 were conference article or other types which were short of full and exact data. One hundred and eightyone full-text articles were reviewed for detailed assessment. Eighteen articles of them were published neither in English or Chinese and 116 trials didn't assess any outcomes relevant to $\beta$-cell function or insulin sensitivity. Twelve articles didn't have the detailed information (change value or $95 \% \mathrm{CI}$ of primary outcome). In 25 trials, agents, such as losartan and fenofibrate, had effect on both SUA and $\beta$-cell function or insulin sensitivity. Finally, 7 eligible citations were included in our meta-analysis ${ }^{26-32}$. Then, manual searching was conducted according to the references of relevant acquired articles. The search was later updated to March 1, 2020. No newly identified study was included in the analyses.

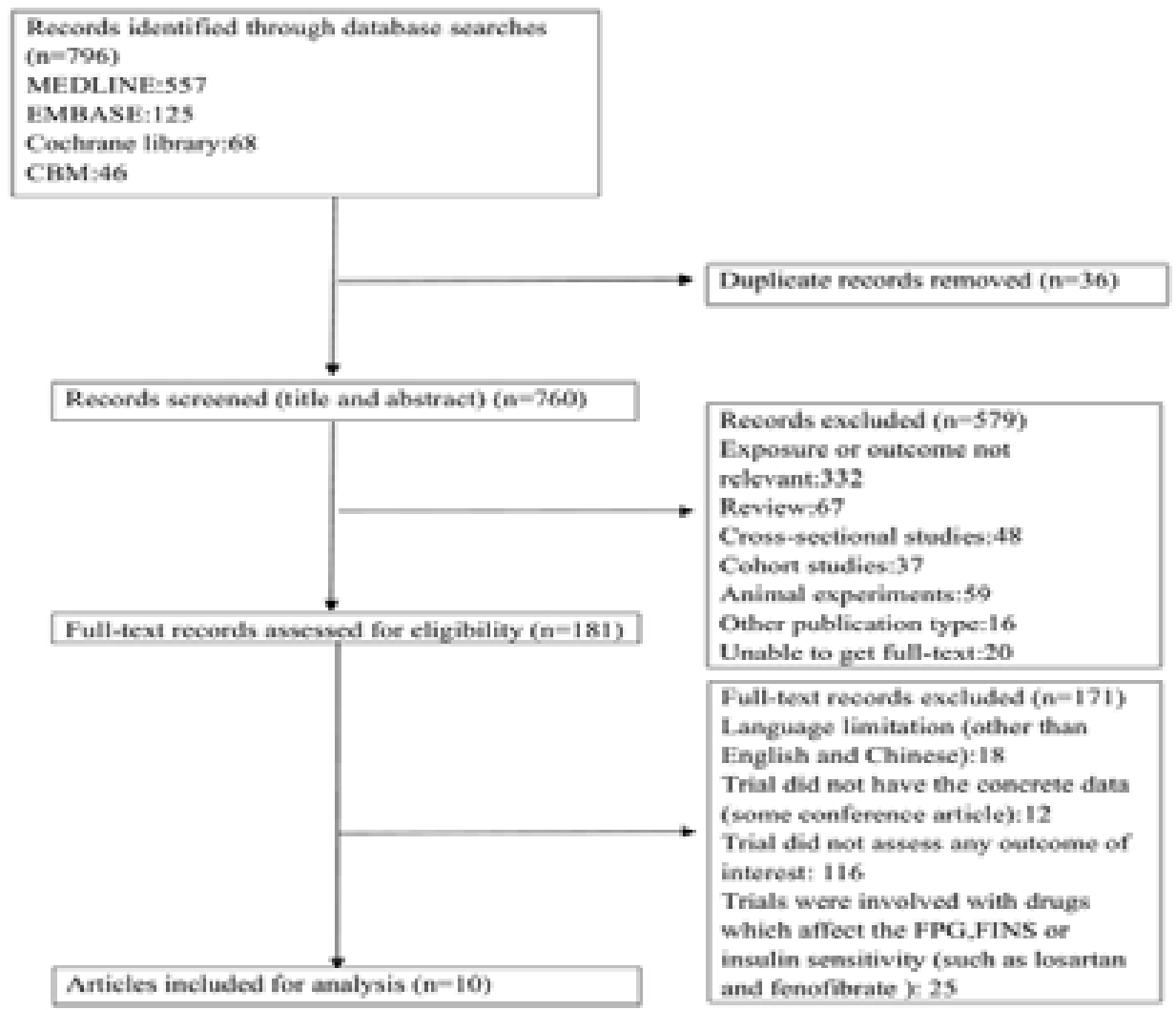

Figure 1. Flowchart of the study selection.

\section{Study characteristics}

The characteristics of the included studies were presented in Table 1. Seven randomized controlled-trials included a total of 503 participants (mean age $50 \pm 10.50$ years) ${ }^{26-32}$. The duration of follow-up period ranged from 2 weeks to 156 weeks, with a median of 60 weeks. Of these, six studies were intervened with allopurinol $^{26-31}$ and one with benzbromarone ${ }^{32}$. All the individual studies were based on general population of predominantly middle-aged or older participants with hyperuricemia, except one based on non-hyperuricemia but induced to hyperuricemia by excessive fructose intake $^{27}$. Then the hyperuricemia patients induced by excessive fructose intake were randomized to two groups: one was given allopurinol when the SUA was greater than $420 \mu \mathrm{mol} / \mathrm{L}$, the other received no treatment. Three of these studies were carried out in the patients of hyperuricemia with either T2DM or impaired glucose tolerance (IGT) ${ }^{26,29,31}$. 
Table 1. Descriptive characteristics of included articles

\begin{tabular}{|c|c|c|c|c|c|c|c|c|}
\hline References & Participants & $\mathrm{n}$ & Age & Duration, design & Intervention & Control & Available Outcomes & Limitation \\
\hline $\begin{array}{l}\text { Szwejkowski et } \\
\text { al [22], } 2013\end{array}$ & $\begin{array}{l}\text { Patients with } \\
\text { hyperuricemia and } \\
\text { T2DM }\end{array}$ & $\begin{array}{l}66(59 \\
\text { completers })\end{array}$ & $\begin{array}{l}64.63 \pm 8.79 \\
\text { years }\end{array}$ & $\begin{array}{l}9 \text { months, } \\
\text { RCT }\end{array}$ & $\begin{array}{l}\text { Allopurinol } 600 \\
\text { mg twice per } \\
\text { day }(n=33)\end{array}$ & Placebo $(n=33)$ & $\begin{array}{l}\text { Difference } 600 \mathrm{mg} \text { allopurinol versus control } \\
(95 \% \mathrm{CI}): \\
\text { UA }(\mu \mathrm{mo} / \mathrm{l}):-251.00[-313.02,-188.98] \\
\text { FPG }(\mathrm{mmol} / \mathrm{l}):-0.43[-2.39,1.53] \\
\text { FINS }(\mu \mathrm{U} / \mathrm{ml}): 4.77[-4.27,13.81]\end{array}$ & $\begin{array}{l}\text { Not adjusted for diabetes duration, kinds } \\
\text { or dosage of hypoglycemic agents. Not } \\
\text { aims to evaluate the effect of uric acid- } \\
\text { lowering therapy on } \beta \text {-cell function or } \\
\text { insulin sensitivity. }\end{array}$ \\
\hline $\begin{array}{l}\text { Perez-Pozo et } \\
\text { al [23], } 2010\end{array}$ & $\begin{array}{l}\text { Participants were } \\
\text { administered } \\
\text { fructose } \\
200 \mathrm{~g} \text { daily }\end{array}$ & $\begin{array}{l}83 \text { ( } 74 \\
\text { completers) }\end{array}$ & $\begin{array}{l}40-65 \text { years } \\
\text { (mean } 51 \pm 1.3 \\
\text { years) }\end{array}$ & 2 weeks, RCT & $\begin{array}{l}\text { Allopurinol } 200 \\
\mathrm{mg} / \mathrm{d}(\mathrm{n}=38)\end{array}$ & $\begin{array}{l}\text { No treatment } \\
(\mathrm{n}=36)\end{array}$ & $\begin{array}{l}\text { Difference 100mg allopurinol versus control } \\
(95 \% \mathrm{CI}) \\
\text { UA }(\mu \mathrm{mo} / \mathrm{l}):-178.00[-182.29,-173.71] \\
\text { FPG }(\mathrm{mmol} / \mathrm{l}): 0.10[0.07,0.13] \\
\text { FINS }(\mu \mathrm{U} / \mathrm{ml}):-0.58[-0.85,-0.31] \\
\text { HOMA-IR : }-0.39[-0.85,0.07]\end{array}$ & $\begin{array}{l}\text { Participates were induced } \\
\text { to hypeluricemia by fructose. Not } \\
\text { adjusted for diabetes duration, kinds or } \\
\text { dosage of hypoglycemic agents. Not } \\
\text { aims to evaluate the effect of uric acid- } \\
\text { lowering therapy on } \beta \text {-cell function or } \\
\text { insulin sensitivity. }\end{array}$ \\
\hline $\begin{array}{l}\text { Takir et al [24], } \\
2014\end{array}$ & $\begin{array}{l}\text { Patients with } \\
\text { hyperuricemia }\end{array}$ & 73 & $\begin{array}{l}50.76 \pm 13.78 \\
\text { years }\end{array}$ & 3 months, RCT & $\begin{array}{l}\text { Allopurinol } 300 \\
\mathrm{mg} / \mathrm{d}(\mathrm{n}=40)\end{array}$ & $\begin{array}{l}\text { No treatment } \\
(\mathrm{n}=33)\end{array}$ & $\begin{array}{l}\text { Difference } 300 \mathrm{mg} \text { allopurinol versus control } \\
(95 \% \mathrm{CI}): \\
\text { UA }(\mu \mathrm{mo} / \mathrm{l}):-72.00[-95.86,-48.14] \\
\text { FPG }(\mathrm{mmol} / \mathrm{l}):-0.40[-0.61,-0.19] \\
\text { FINS }(\mu \mathrm{U} / \mathrm{ml}):-2.00[-3.99,-0.01] \\
\text { HOMA-IR : }-3.40[-5.22,-1.58]\end{array}$ & $\begin{array}{l}\text { Choice of allopurinol versus control was } \\
\text { performed by the treating physician. } \\
\text { Hence, it was not a pure randomization. }\end{array}$ \\
\hline $\begin{array}{l}\text { Liu et al [25], } \\
2015\end{array}$ & $\begin{array}{l}\text { Patients } \\
\text { with T2DM and } \\
\text { hyperuricemia }\end{array}$ & $\begin{array}{l}176 \text { (152 } \\
\text { completers) }\end{array}$ & $\begin{array}{l}50.5 \pm \\
10.49 \text { years }\end{array}$ & 3 years, RCT & $\begin{array}{l}\text { Allopurinol } \\
\text { (starting from } \\
100 \mathrm{mg} / \text { day) } \\
\text { adjust to SUA } \\
(\mathrm{n}=88)\end{array}$ & $\begin{array}{l}\text { No } \\
\text { treatment when } \\
\text { the SUA was } \\
\text { less than } \\
476 \mu \mathrm{mol} / \mathrm{L} \\
(\mathrm{n}=88)\end{array}$ & $\begin{array}{l}\text { Difference allopurinol versus } \\
\text { control }(95 \% \mathrm{CI}) \\
\text { UA }(\mu \mathrm{mo} / \mathrm{l}):-138.00[-142.13,-133.87] \\
\text { FPG }(\mathrm{mmol} / \mathrm{l}): 0.01[-0.11,0.13] \\
\text { FINS }(\mu \mathrm{U} / \mathrm{ml}):-0.51[-0.73,-0.29] \\
\text { HOMA-IR : }-0.67[-0.99,-0.34]\end{array}$ & $\begin{array}{l}\text { Open-label design and the lack of a } \\
\text { placebo control. Not adjusted } \\
\text { for diabetes duration, kinds or dosage } \\
\text { of hypoglycemic agents. Not aims to } \\
\text { evaluate the effect of uric acid-lowering } \\
\text { therapy on } \beta \text {-cell function or insulin } \\
\text { sensitivity. }\end{array}$ \\
\hline $\begin{array}{l}\text { Ding et al [26], } \\
2012\end{array}$ & $\begin{array}{l}\text { Patients with } \\
\text { hyperuricemia }\end{array}$ & 60 & $48.0 \pm 11.7$ years & $\begin{array}{l}3 \sim 8 \text { months } \\
\text { (mean } 4.3 \\
\text { months), RCT }\end{array}$ & $\begin{array}{l}\text { Allopurinol } 100 \\
\text { mg twice a day } \\
(\mathrm{n}=30)\end{array}$ & $\begin{array}{l}\text { No treatment } \\
(\mathrm{n}=30)\end{array}$ & $\begin{array}{l}\text { Difference } 200 \mathrm{mg} \text { allopurinol versus control } \\
(95 \% \mathrm{CI}): \\
\text { UA }(\mu \mathrm{mo} / \mathrm{l}):-140.6[-2.63,-1.69] \\
\text { FPG }(\mathrm{mmol} / \mathrm{l}): 0.17[-0.17,0.51] \\
\text { FINS }(\mu \mathrm{U} / \mathrm{ml}): 7.28[5.62,8.94] \\
\text { HOMA-IR: } 0.16[-0.34,0.67]\end{array}$ & $\begin{array}{l}\text { Unclear for random sequence } \\
\text { generation or double-blind design. Not } \\
\text { adjusted for kinds or dosage of } \\
\text { hypoglycemic agents. }\end{array}$ \\
\hline $\begin{array}{l}\text { Le et al [27], } \\
2013\end{array}$ & $\begin{array}{l}\text { Patients with } \\
\text { hyperuricemia and } \\
\text { IGT }\end{array}$ & 40 & $\begin{array}{l}44.65 \pm 2.27 \\
\text { years }\end{array}$ & 6 months, RCT & $\begin{array}{l}\text { allopurinol } 100 \\
\text { mg three times } \\
\text { a day }(n=20)\end{array}$ & $\begin{array}{l}\text { Low purine and } \\
\text { diabetes diet } \\
(\mathrm{n}=20)\end{array}$ & $\begin{array}{l}\text { Difference } 300 \mathrm{mg} \text { allopurinol versus } \\
\text { control }(95 \% \mathrm{CI}) \\
\text { UA }(\mu \mathrm{mo} / \mathrm{l}):-203.00[-221.97,-184.03] \\
\text { FPG }(\mathrm{mmol} / \mathrm{l}):-0.66[-0.86,-0.46] \\
\text { FINS }(\mu \mathrm{U} / \mathrm{ml}):-10.56[-13.39,-7.73] \\
\text { HOMA-IR : }-3.56[-4.59,-2.53]\end{array}$ & $\begin{array}{l}\text { Unclear for random sequence } \\
\text { generation or double-blind design. Not } \\
\text { adjusted for kinds or dosage of } \\
\text { hypoglycemic agents. }\end{array}$ \\
\hline $\begin{array}{l}\text { Ogino al [28], } \\
2016\end{array}$ & $\begin{array}{l}\text { Patients with } \\
\text { hyperuricemia }\end{array}$ & 14 & $60 \pm 5$ years & $\begin{array}{l}8 \text { weeks, } \\
\text { randomized } \\
\text { crossover } \\
\text { study }\end{array}$ & $\begin{array}{l}\text { Benzbromarone } \\
50 \mathrm{mg} / \mathrm{d}(\mathrm{n}=7)\end{array}$ & Placebo $(\mathrm{n}=7)$ & $\begin{array}{l}\text { Difference } 50 \mathrm{mg} \text { benzbromarone versus } \\
\text { control }(95 \% \mathrm{CI}) \text { : } \\
\text { UA }(\mu \mathrm{mo} / \mathrm{l}):-128.90[-156.88,-100.92] \\
\text { FPG }(\mathrm{mmol} / \mathrm{l}):-0.33[-0.56,-0.10] \\
\text { FINS }(\mu \mathrm{U} / \mathrm{ml}):-7.80[-9.26,-6.34] \\
\text { HOMA-IR : }-1.22[-1.72,-0.72]\end{array}$ & $\begin{array}{l}\text { Participates are patients with CHF and } \\
\text { under the treatment of ACEIs, which } \\
\text { may affect insulin sensitivity. Excluded } \\
\text { diabetes mellitus and antidiabetic } \\
\text { therapy. Short duration and small } \\
\text { sample size. Not aims to evaluate the } \\
\text { effect of uric acid-lowering therapy on } \\
\beta \text {-cell function or insulin sensitivity. }\end{array}$ \\
\hline
\end{tabular}

Abbreviations: T2DM, Type 2 diabetes mellitus; RCT, randomized controlled trial; UA, uric acid; FPG. fasting plasma glucose; FINS, fasting insulin; HOMA-IR, homeostasis model assessment of insulin resistance; IGT, impaired glucose tolerance; CHF, chronic heart failure; ACEIs, angiotensin converting enzyme inhibitors.

\section{Quality of studies}

The quality of the individual studies was assessed by the Cochrane Risk of Bias tool. Details of risk of bias as- sessment are shown in Table S1. The reporting quality was rated as 'high' in three of the studies ${ }^{26,29,32}$, 'medium' in one study ${ }^{27}$, and 'low' in three studies ${ }^{28,30,31}$.

Table S1. Quality assessment of included studies.

\begin{tabular}{|c|c|c|c|c|c|c|c|c|c|}
\hline \multirow[t]{2}{*}{ Study } & \multirow{2}{*}{$\begin{array}{l}\text { Random } \\
\text { sequence } \\
\text { generation }\end{array}$} & \multirow{2}{*}{$\begin{array}{l}\text { Allocation } \\
\text { concealment }\end{array}$} & \multicolumn{3}{|c|}{ Blinding } & \multirow{2}{*}{$\begin{array}{l}\text { Incomplete } \\
\text { outcome data } \\
\text { addressed }\end{array}$} & \multirow{2}{*}{$\begin{array}{l}\text { Free of } \\
\text { selective } \\
\text { reporting }\end{array}$} & \multirow{2}{*}{$\begin{array}{l}\text { Other sources } \\
\text { of bias }\end{array}$} & \multirow[t]{2}{*}{ Quantity } \\
\hline & & & Participants & Personnel & Outcome assessors & & & & \\
\hline $\begin{array}{l}\text { Szwejkowskiet al [22], } \\
2013\end{array}$ & Yes & Yes & Yes & Yes & Yes & Yes & Yes & Not reported & High \\
\hline $\begin{array}{l}\text { Perez-Pozo et al [23], } \\
2010\end{array}$ & Yes & Yes & Yes & No & Not reported & Yes & Yes & Not reported & Medium \\
\hline Takir et al [24], 2014 & Unclear & Unclear & Unclear & Unclear & Not reported & Yes & Yes & Not reported & Low \\
\hline Liu et al [25], 2015 & Yes & Yes & Yes & Yes & Yes & Yes & Yes & Not reported & High \\
\hline Ding et al [26], 2012 & Unclear & Unclear & Unclear & Unclear & Not reported & Unclear & Yes & Not reported & Low \\
\hline Le et al [27], 2013 & Unclear & Unclear & Unclear & Unclear & Not reported & Yes & Yes & Not reported & Low \\
\hline Ogino et al [28], 2016 & Yes & Yes & Yes & Yes & Yes & Yes & Yes & Not reported & High \\
\hline
\end{tabular}

\section{Outcomes}

FPG, FINS and HOMA- $\beta$ (homeostasis model assessment of $\beta$-cell function). Seven studies26-32 with 503 participants in our main analyses for the changes in FPG and FINS from baseline pooled estimates showed hypouricemic treatment significantly decreased SUA 
(WMD, $-165.52 \mu \mathrm{mol} / \mathrm{L}, 95 \%$ CI -189.91 to -141.14 ) by using a random effects model. At the same time, FINS was reduced by $-1.43 \mu \mathrm{IU} / \mathrm{ml}$ (WMD, 95\% CI -2.78 to -0.09) (Figure 2A). There were significant heterogeneities for the analyses of SUA $\left(\mathrm{I}^{2}=98 \%\right.$; $\left.\mathrm{P}=0.00\right)$ and FINS $\left(I^{2}=97.4 \% ; P=0.00\right)$. However, hypouricemic therapy had no significant effect on FPG (WMD $-0.19 \mathrm{mmol} / \mathrm{L}, 95 \% \mathrm{CI}-0.42$ to 0.05 ) (Figure $2 \mathrm{~B}$ ). Two studies with 236 participates measured HOMA- $\beta$ and reported data that could be pooled in the analysis ${ }^{29,30}$.
Compared with the controls, there was no statistically significant change in HOMA- $\beta$ (WMD - 0.02, 95\% CI -0.28 to 0.24 ) (Figure 2C). Moderate heterogeneity was present between the two studies $\left(\mathrm{I}^{2}=40.4 \% ; \mathrm{P}=0.20\right)$. Insulin sensitivity (HOMA-IR) Six studies with 437 participants reported the change in HOMA-IR from baseline ${ }^{27-32}$. The treatment significantly mitigated HOMA-IR (WMD -0.65, 95\%CI -1.05 to -0.24) (Figure 2D). Heterogeneity of the effect measures on HOMA-IR was detected $\left(\mathrm{I}^{2}=96.6 \% ; \mathrm{P}=0.00\right)$.

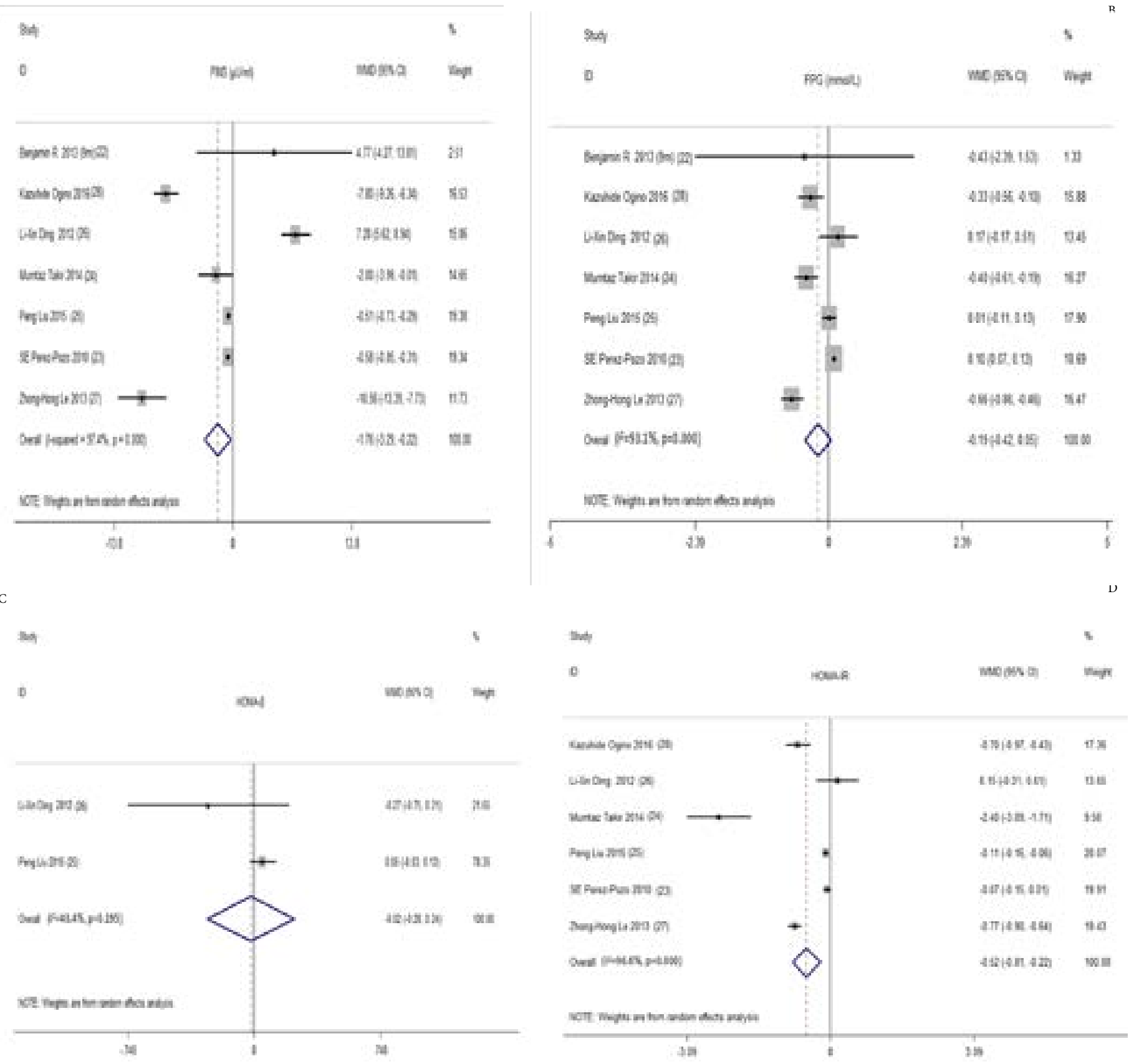

Figure 2. Forest plot and weighted mean differences (WMD) for effect of the uric acid lowering therapy on FINS (A), FPG (B), HOMA- $\beta$ (C) and HOMA-IR (D). Abbreviations: Szwejkowski-1 is the result of 6 months' follow up. Szwejkowski-2 is the result of 9 months' follow up. WMD, weighted mean differences; CI, confidence interval; FINS, fasting insulin; HOMA- $\beta$, homeostasis model assessment of $\beta$-cell function index; HOMA-IR, homeostasis model assessment of insulin resistance.

Cardiovascular risk factors (BP, TC and TG) Four studies involving 376 participants demonstrated that ULT was associated with a significant reduction of systolic blood pressures (SBP) (WMD -2.45 mm Hg, 95\%CI
-4.57 to -0.33 ) (Figure 3A) and diastolic blood pressures (DBP) (WMD -3.41 mm Hg, 95\%CI -3.87 to -2.95)

( Figure 3B) 26, 27, 29, 30. There was high level of heterogeneity for the analysis of SBP $\left(\mathrm{I}^{2}=81.2 \%\right.$; $\mathrm{P}=$ 
$0.00)$, but no heterogeneity for DBP $\left(\mathrm{I}^{2}=0.00 \%\right.$; $\mathrm{P}=$ $0.50)$.

With regard to serum lipids, changes were pooled for 4 studies with 376 participants $28,29,30,32$. It suggested that the hyperuricemia therapy had no significant

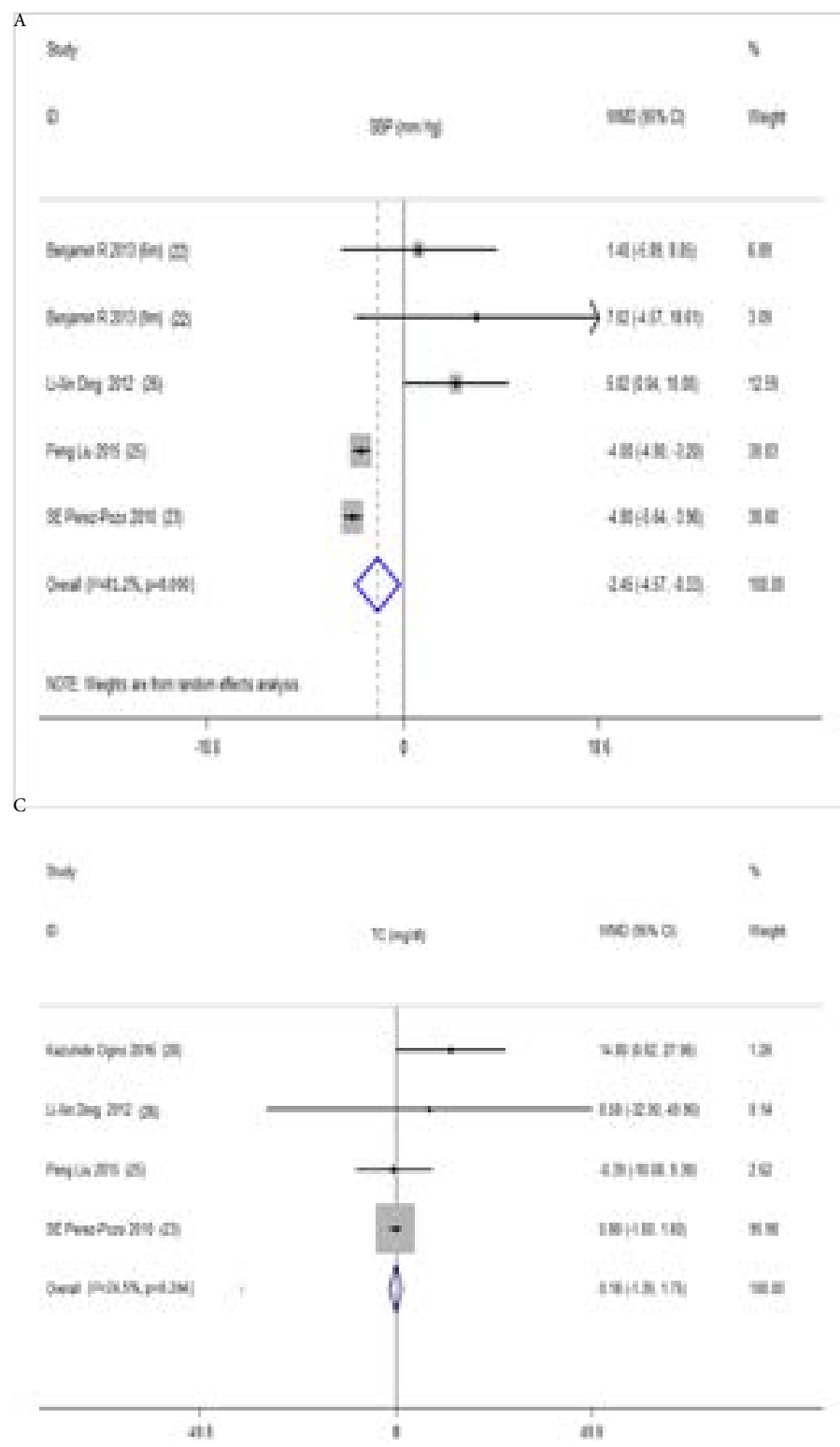

reduction in TC (WMD $1.48 \mathrm{mg} / \mathrm{dl}, 95 \%$ CI -3.34 to 6.39) (Figure 3C) and TG (WMD $3.15 \mathrm{mg} / \mathrm{dl}, 95 \% \mathrm{CI}$ -9.83 to 16.14) (Figure 3D) compared with the control. Low level of heterogeneity was observed in the analysis of TC $\left(\mathrm{I}^{2}=24.5 \% ; \mathrm{P}=0.26\right)$. But the heterogeneity for the analysis of TG was severe $\left(\mathrm{I}^{2}=93.5 \% ; \mathrm{P}=0.00\right)$.
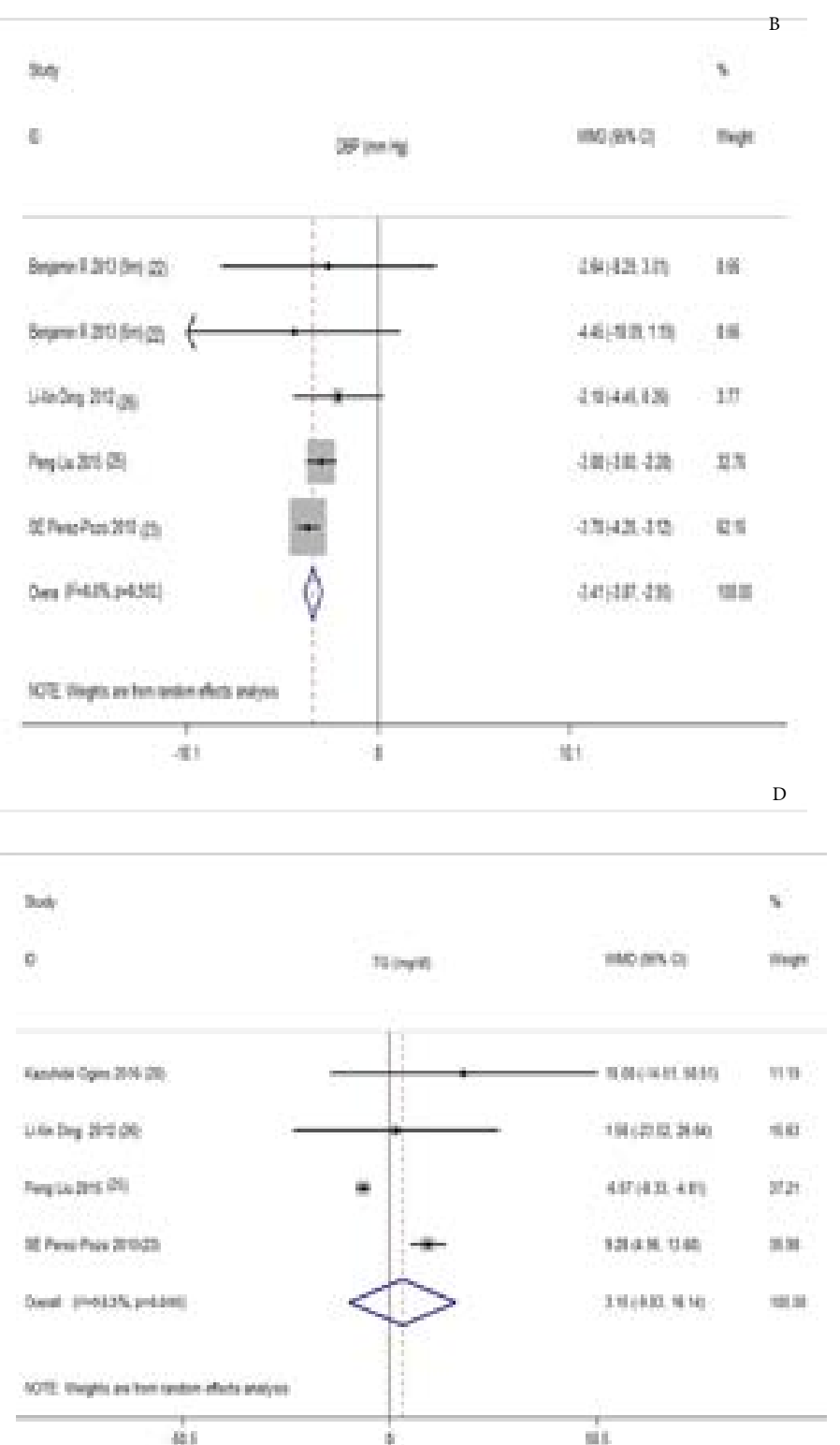

Figure 3. Forest plot and weighted mean differences (WMD) for effect of the uric acid lowering therapy on SBP (A), DBP (B), TC (C) and TG (D). Abbreviations: Szwejkowski-1 is the result of 6 months' follow up. Szwejkowski-2 is the result of 9 months' follow up; WMD, weighted mean differences; CI, confidence interval; SBP, systolic blood pressure; DBP, diastolic blood pressure; TC, total cholesterol; TG, triglyceride.

\section{Subgroup and sensitivity analyses}

Significant heterogeneities were observed in the primary outcome of FPG, FINS and HOMA-IR, therefore we performed further analyses. The subgroup analyses were stratified by (1) participates with or without hyperglycemia (normal glucose regulation, impaired glucose tolerance or diabetes mellitus), (2) dosage of hypouricemic agents (allopurinol $\geq 300 \mathrm{mg} / \mathrm{d}$, allopurinol $<300$ $\mathrm{mg} / \mathrm{d}$, benzbromarone $50 \mathrm{mg} / \mathrm{d}$ or adjust according to
SUA), (3) mean UA at baseline ( $\geq 420 \mathrm{umol} / \mathrm{L}$ or $<420$ umol/L), (4) reduction of SUA ( $\geq 150 \mathrm{umol} / \mathrm{L}$ or $<$ $150 \mathrm{umol} / \mathrm{L}$ ), (4) mean age of participants (mean age $<50$ years or mean age $<50$ years), (4) duration of the study (duration $\geq 6$ months or duration $<6$ months), (5) body mass index (BMI) ( $\geq 28 \mathrm{~kg} / \mathrm{m}^{2}$ or $<28 \mathrm{~kg} /$ $\mathrm{m}^{2}$ ), (6) the number of participants $(\mathrm{n}>60$ or $\mathrm{n} 60$ ) (Table S2-S4). Because of only 2 studies with data of HOMA- $\beta$, we didn't perform subgroup or sensitivity analysis.

African Health Sciences, Vol 21 Issue 1, March, 2021 
Table S2. Subgroup analysis for FPG of various variables.

\begin{tabular}{|c|c|c|c|c|}
\hline Group & Number of studies & WMD $(95 \% \mathrm{CI})$ & P for heterogeneity & $\mathrm{I}^{2} \%$ \\
\hline Total & 7 & $-0.19[-0.42,0.05]$ & $<0.001$ & 93.00 \\
\hline PG & & & & 94.00 \\
\hline NGR & 4 & $-0.33[-0.95,0.29]$ & $<0.001$ & 93.70 \\
\hline IGT or DM & 3 & $-0.12[-0.42,0.19]$ & $<0.001$ & 91.20 \\
\hline \multicolumn{5}{|l|}{ Dosage } \\
\hline Allopurinol $\geq 300 \mathrm{mg} / \mathrm{d}$ & 3 & $-0.53[-0.74,-0.32]$ & 0.217 & 34.60 \\
\hline Allopurinol $<300 \mathrm{mg} / \mathrm{d}$ & 2 & $0.10[0.07,0.13]$ & 0.688 & 0 \\
\hline Benzbromarone $50 \mathrm{mg} / \mathrm{d}$ & 1 & $-0.33[-0.56,-0.10]$ & - & - \\
\hline Adjust according to SUA & 1 & $0.01[-0.11,0.13]$ & - & - \\
\hline \multicolumn{5}{|l|}{ Mean SUA at baseline } \\
\hline$\geq 420 \mathrm{umol} / \mathrm{L}$ & 4 & $-0.17[-0.61,0.27]$ & $<0.001$ & 92.00 \\
\hline$<420 \mathrm{umol} / \mathrm{L}$ & 3 & $-0.20[-0.58,0.18]$ & $<0.001$ & 94.00 \\
\hline \multicolumn{5}{|l|}{ Reduction of SUA } \\
\hline$\geq 150 \mathrm{umol} / \mathrm{L}$ & 3 & $-0.29[-0.99,0.41]$ & $<0.001$ & 96.00 \\
\hline$<150 \mathrm{umol} / \mathrm{L}$ & 4 & $-0.13[-0.38,0.11]$ & $<0.001$ & 85.00 \\
\hline \multicolumn{5}{|l|}{ Mean age } \\
\hline$\geq 50$ years & 5 & $-0.13[-0.34,0.07]$ & $<0.001$ & 88.80 \\
\hline$<50$ years & 2 & $-0.26[-1.07,0.56]$ & $<0.001$ & 94.10 \\
\hline \multicolumn{5}{|l|}{ BMI } \\
\hline$\geq 28$ & 3 & $-0.15[-0.62,0.31]$ & $<0.001$ & 91.00 \\
\hline$<28$ & 3 & $-0.05[-0.29,0.20]$ & $<0.001$ & 99.00 \\
\hline \multicolumn{5}{|l|}{ Duration } \\
\hline$\geq 6$ months & 3 & $-0.33[-0.95,0.29]$ & $<0.001$ & 93.70 \\
\hline$<6$ months & 4. & $-0.12[-0.42,0.19]$ & $<0.001$ & 91.20 \\
\hline \multicolumn{5}{|l|}{ Number of participant } \\
\hline$>60$ & 4 & $-0.07[-0.28,0.13]$ & $<0.001$ & 87.10 \\
\hline$\leq 60$ & 3 & $-0.29[-0.72,0.14]$ & $<0.001$ & 88.60 \\
\hline
\end{tabular}

Abbreviations: PG, plasma glucose; NGR, normal glucose regulation; IGT, impaired glucose tolerance; DM, diabetes mellitus; SUA, serum uric acid; BMI, body mass index.

Table S3. Subgroup analysis for FINS of various variables.

\begin{tabular}{|c|c|c|c|c|}
\hline Group & Number of studies & WMD (95\% CI) & P for heterogeneity & $\mathrm{I}^{2} \%$ \\
\hline Total & 7 & $-1.43[-2.78,-0.09]$ & $<0.001$ & 97.00 \\
\hline \multicolumn{5}{|c|}{$1.10[-10,0,0.09]$} \\
\hline NGR & 4 & $-0.65[-3.86,2.56]$ & $<0.001$ & 98.00 \\
\hline IGT or DM & 3 & $-2.79[-10.92,5.34]$ & $<0.001$ & 96.00 \\
\hline \multicolumn{5}{|l|}{ Dosage } \\
\hline Allopurinol $\geq 300 \mathrm{mg} / \mathrm{d}$ & 3 & $-3.52[-10.68,3.64]$ & $<0.001$ & 94.00 \\
\hline Allopurinol < $300 \mathrm{mg} / \mathrm{d}$ & 2 & $3.31[-4.40,11.01]$ & $<0.001$ & 99.00 \\
\hline benzbromarone $50 \mathrm{mg} / \mathrm{d}$ & 1 & $-7.80[-10.31,-5.29]$ & - & - \\
\hline Adjust according to SUA & 1 & $-0.51[-0.73,-0.29]$ & - & - \\
\hline \multicolumn{5}{|l|}{ Mean SUA at baseline } \\
\hline$\geq 420 \mathrm{umol} / \mathrm{L}$ & 4 & $0.35[-13.48,14.17]$ & $<0.001$ & 90.00 \\
\hline$<420 \mathrm{umol} / \mathrm{L}$ & 3 & $-1.27[-2.02,-0.52]$ & $<0.001$ & 98.00 \\
\hline \multicolumn{5}{|l|}{ Reduction of SUA } \\
\hline$\geq 150 \mathrm{umol} / \mathrm{L}$ & 3 & $-2.82[-10.90,5.26]$ & $<0.001$ & 96.00 \\
\hline$<150 \mathrm{umol} / \mathrm{L}$ & 4 & $-0.49[-3.53,2.55]$ & $<0.001$ & 97.00 \\
\hline \multicolumn{5}{|l|}{ Mean age } \\
\hline$\geq 50$ years & 5 & $-1.47[-2.31,-0.63]$ & $<0.001$ & 91.00 \\
\hline$<50$ years & 2 & $-1.60[-19.08,15.88]$ & $<0.001$ & 99.00 \\
\hline \multicolumn{5}{|l|}{ BMI } \\
\hline$\geq 28$ & 3 & $-1.09[-2.49,0.30]$ & 0.005 & 81.00 \\
\hline$<28$ & 3 & $-0.20[-6.43,6.03]$ & $<0.001$ & 98.00 \\
\hline \multicolumn{5}{|l|}{ Duration } \\
\hline$\geq 6$ months & 3 & $-2.79[-10.92,5.34]$ & $<0.001$ & 96.00 \\
\hline$<6$ months & 4. & $-0.65[-3.86,2.56]$ & $<0.001$ & 98.00 \\
\hline \multicolumn{5}{|l|}{ Number of participant } \\
\hline$>60$ & 4 & $-0.77[-1.23,-0.32]$ & $<0.001$ & 73.00 \\
\hline$\leq 60$ & 3 & $-3.65[-15.67,8.36]$ & $<0.001$ & 99.00 \\
\hline
\end{tabular}

Abbreviations: PG, plasma glucose; NGR, normal glucose regulation; IGT, impaired glucose tolerance; DM, diabetes mellitus; SUA, serum uric acid; BMI, body mass index. 
Table S4. Subgroup analysis for HOMA-IR of various variables.

\begin{tabular}{|c|c|c|c|c|}
\hline Group & Number of studies & WMD (95\% CI) & P for heterogeneity & $\mathrm{I}^{2} \%$ \\
\hline Total & 6 & $-0.65[-1.05,-0.24]$ & $<0.001$ & 98.00 \\
\hline \multicolumn{5}{|l|}{ PG } \\
\hline NGR & 4 & $-0.76[-2.02,0.51]$ & $<0.001$ & 99.00 \\
\hline IGT or DM & 2 & $-0.44[-1.08,0.21]$ & $<0.001$ & 99.00 \\
\hline \multicolumn{5}{|l|}{ Dosage } \\
\hline Allopurinol $\geq 300 \mathrm{mg} / \mathrm{d}$ & 2 & $-1.58[-3.18,0.02]$ & $<0.001$ & 99.00 \\
\hline Allopurinol $<300 \mathrm{mg} / \mathrm{d}$ & 2 & $-0.06[-0.14,0.02]$ & 0.351 & 0 \\
\hline benzbromarone $50 \mathrm{mg} / \mathrm{d}$ & 1 & $-0.70[-1.30,-0.10]$ & - & - \\
\hline Adjust according to SUA & 1 & $-0.11[-0.16,-0.06]$ & - & - \\
\hline \multicolumn{5}{|l|}{ Mean SUA at baseline } \\
\hline$\geq 420 \mathrm{umol} / \mathrm{L}$ & 3 & $-2.21[-5.10,0.69]$ & $<0.001$ & 96.00 \\
\hline$<420 \mathrm{umol} / \mathrm{L}$ & 3 & $-0.77[-1.06,-0.47]$ & 0.090 & 59.00 \\
\hline \multicolumn{5}{|l|}{ Reduction of SUA } \\
\hline$\geq 150 \mathrm{umol} / \mathrm{L}$ & 2 & $-1.83[-4.58,0.91]$ & 0.003 & 89.00 \\
\hline$<150 \mathrm{umol} / \mathrm{L}$ & 4 & $-1.21[-2.16,-0.27]$ & $<0.001$ & 93.00 \\
\hline \multicolumn{5}{|l|}{ Mean age } \\
\hline$\geq 50$ years & 4 & $-0.79[-1.29,-0.29]$ & $<0.001$ & 99.00 \\
\hline$<50$ years & 2 & $-0.34[-1.24,0.56]$ & $<0.001$ & 93.10 \\
\hline \multicolumn{5}{|l|}{ BMI } \\
\hline$\geq 28$ & 2 & $-0.64[-0.83,-0.45]$ & 0.540 & 0 \\
\hline$<28$ & 3 & $-1.48[-3.17,0.21]$ & $<0.001$ & 95.00 \\
\hline \multicolumn{5}{|l|}{ Duration } \\
\hline$\geq 6$ months & 2 & $-0.44[-1.08,0.21]$ & $<0.001$ & 95.20 \\
\hline$<6$ months & 4 & $-0.76[-2.02,0.51]$ & $<0.001$ & 98.80 \\
\hline \multicolumn{5}{|l|}{ Number of participant } \\
\hline$>60$ & 3 & $-0.81[-1.37,-0.25]$ & $<0.001$ & 85.20 \\
\hline$\leq 60$ & 3 & $-0.45[-1.05,0.15]$ & $<0.001$ & 95.30 \\
\hline
\end{tabular}

Abbreviations: PG, plasma glucose; NGR, normal glucose regulation; IGT, impaired glucose tolerance; DM, diabetes mellitus; SUA, serum uric acid; BMI, body mass index.

In our analysis, we found that (1) FPG was significantly decreased in high dosage group (allopurinol $\geq 300$ $\mathrm{mg} / \mathrm{d}$ ) (WMD $-0.53 \mathrm{mmol} / \mathrm{L}, 95 \% \mathrm{CI}-0.74$ to -0.32 ), while there was a minimal but statistically increase in the group of allopurinol $<300 \mathrm{mg} / \mathrm{d}(0.10 \mathrm{mmol} / \mathrm{L}$; $95 \% \mathrm{CI}$ 0.07 to 0.13 ). Moreover, the heterogeneities were significant decreased among studies in group of allopurinol $\geq 300 \mathrm{mg} / \mathrm{d}\left(\mathrm{I}^{2}=34.6 \% ; \mathrm{P}=0.22\right)$ as well as in group of allopurinol $<300 \mathrm{mg} / \mathrm{d}\left(\mathrm{I}^{2}=0 \% ; \mathrm{P}=0.69\right)$ (Table $3)$; (2) the reductions of FINS were more significant in older group (mean age $\geq 50$ years) (WMD $-1.47 \mu \mathrm{U} /$ $\mathrm{ml} ; 95 \% \mathrm{CI}-2,31$ to -0.63$)$ and in the large number of participates group (WMD $-0.77 \mu \mathrm{U} / \mathrm{ml} ; 95 \% \mathrm{CI}-1.23$ to -0.32) (Table S3); (3) the reduction of HOMA-IR was more remarkable in the patients with normal blood glucose (WMD -0.68; 95\%CI -1.33 to -0.02), lower UA levels at baseline (WMD -0.77; 95\%CI -1.06 to -0.47 ), older age (WMD -0.79; 95\%CI -1.29 to -0.29), higher $\mathrm{BMI}$ at baseline (WMD -0.64; 95\%CI -0.83 to -0.45 ), duration of treatment less than 6 months (WMD -0.81;
$95 \% \mathrm{Cl}-1.37$ to -0.25$)$ and in the trails with large number sample size (WMD $-0.81 ; 95 \% \mathrm{Cl}-1.37$ to -0.25 ). Meanwhile, the heterogeneity ( $\mathrm{P}=0.35$ ) of HOMA-IR was deceased among studies in group of allopurinol $<$ $300 \mathrm{mg} / \mathrm{d}$ (Table S4); (4) no significant difference of any other outcome between other subgroups were observed; (5) the heterogeneities were decreased for studies of FPG and HOMA-IR in different dosage groups, studies for HOMA-IR in BMI $\geq 28 \mathrm{~kg} / \mathrm{m}^{2}$ and with mean UA at baseline $<420 \mathrm{umol} / \mathrm{L}$; (6) the improvement of HOMA-IR was more significant in individuals with lower UA or get higher BMI at baseline. In the sensitivity analyses, omission of any individual study from the meta-analysis did not significantly alter the pooled effects or heterogeneities.

\section{Publication bias}

We found no evidence of substantial publication bias from Egger's or Begg's regression test $(\mathrm{P}>0.05)$ for any outcome examined (Table S5). 
Table S5. Evaluation of publication bias for studies included in the meta-analysis

\begin{tabular}{lll}
\hline & P value of the Begg's test & P value of the Fgger's test \\
\hline Primary outcomes & & \\
FPG $(\mathrm{mmol} / \mathrm{l})$ & 1.000 & 0.079 \\
FINS $(\mu \mathrm{U} / \mathrm{ml})$ & 0.764 & 0.638 \\
HOMA-IR & 0.260 & 0.176 \\
Secondary outcomes & & \\
SBP $(\mathrm{mm} \mathrm{Hg})$ & 0.462 & 0.056 \\
DBP $(\mathrm{mm} \mathrm{Hg})$ & 1.000 & 0.523 \\
TG $(\mathrm{mg} / \mathrm{dl})$ & 0.734 & 0.448 \\
TC $(\mathrm{mg} / \mathrm{dl})$ & 0.308 & 0.343 \\
\hline
\end{tabular}

Abbreviations: FPG, fasting plasma glucose; FINS, fasting insulin; HOMA-IR, homeostasis model assessment of insulin resistance; SBP, systolic blood pressure; DBP, diastolic blood pressure; TG, triglyceride; TC, total cholesterol.

\section{Discussion}

In metabolic syndrome, obesity and type 2 diabetes, the patients usually have metabolic inflammation, insulin resistance and high uric acid. Previous studies showed that insulin resistance is associated with high uric acid ${ }^{6}$, ${ }^{33}$. In this meta-analysis, we showed that ULT decreased HOMA-IR, FINS and BP, but had no effect on FPG, HOMA- $\beta$ or serum lipids. Of note, further stratified analyses indicated that high dosage of uric acid-lowering agents decreased FPG. These results indicate that uric acid lowering treatment may improve glucose metabolism.

In this present study, we demonstrated that hypouricemic therapy could improve insulin resistance in patients with hyperuricemia. It has been reported that hyperuricemia is an independent risk of T2DM and closely associated with IR in observational studies $9,10,14,19$, ${ }^{34}$ as well as meta-analyses ${ }^{10,34}$. Serum UA is an indirect reflection of intracellular urate, which is postulated to be the direct cause of IR. ${ }^{35}$ Several mechanisms might account for it. Animal experiments showed that 4 week-treatment with allopurinol in rats with hyperuricemia induced by fructose-feeding restored insulin sensitivity significantly ${ }^{36}$. It had been reported that high uric acid (HUA) induces IR by inhibiting insulin signaling, including inhibition of phosphorylatipn of Akt (Ser473) response to insulin and increased phosphor-insulin receptor substrate 1 (Ser307). This effect may be mediated by the generation of abnormal amounts of reactive oxygen species (ROS), as antioxidant $\mathrm{N}$-acetylcysteine blocked HUA-induced activation of insulin receptor substrate 1 (IRS-1) and inhibition of Akt phosphorylation ${ }^{3,6}$. Furthermore, a recent study also reported that elevated serum xanthine oxidase activity, but not UA cncentration, was associated with an increased risk of developing $\mathrm{T}^{2} \mathrm{DM}^{37}$, and xanthine oxidase inhibition reduced inflammatory adipokine levels and improved stress-induced insulin sensitivity ${ }^{38}$. Meng et al. also showed that IR was higher in primary gout patients as compared with normal individuals, and was mitigated after 12 week-hypouricemic treatment ${ }^{39}$. It is interesting to note that the stratified analyses showed that the reduction of IR was more remarkable in the patients with older age and in the trails with large sample size and normal blood glucose, as well as in patients received the less duration of treatment in our study. It has been reported that serum levels of uric acid were increased with age $e^{9,40-44}$ and hyperuricemic patients usually company with relatively higher central obesity, serum lipids and BP, which were closely associated with $\operatorname{IR}^{14,15,42,45,46}$. Hence, IR reduction by hypouricemic treatment in patients with older age could be more significant as it was reported older patients who received anti-hyperuricemic agent were more likely to reach SUA goal $^{47}$. Dose-response analysis showed the risk of type 2 diabetes was increased by $6 \%$ per $1 \mathrm{mg} / \mathrm{dl}$ increment in SUA level ${ }^{10}$. Hare et al. showed that patients with higher SUA levels benefited more after receiving uric acid lowering treatment, and post hoc analysis showed that the possible reason is that patients with high uric acid group received moe significant reductions in uric acid after treatment ${ }^{48,49}$. IR was associated with various conditions such as obesity, inflammation, mitochondrial dysfunction, hyperinsulinemia and lipotoxicity/ hyperlipidemi $a^{50,51}$. It is obvious that the hyperuricemic patients with normal glucose levels had less risk factors for IR than those with IGT and diabetes, hypouricemic treatment therefore was more effective in improvement of IR in the patients with normal glucose than in those with IGT or diabetes as indicated in our study. We also showed the reduction of IR was more significant in patients received less duration of treatment. Actually, all these 4 studies with less duration of treatment were conducted in hyperuricemic patients with normal glucose, and this is why the reduction of IR was more significant in the patients. 
Our study showed that FINS was significantly reduced with SUA and subgroup analyses revealed that FINS was further reduced in older individuals and in the trials with the large sample size. These results suggest that ULT improves insulin sensitivity, which is agreeable with above results. Actually, Meng et al. also found that significant higher levels of FINS and HOMA-IR in gout patients than those in the controls, but no significant difference was found regarding FPG $^{39}$. Although FPG didn't be significantly reduced in our primary analysis, stratified analyses showed that FPG were decreased in the trials with allopurinol $\geq 300 \mathrm{mg} / \mathrm{d}$ and these trials, actually, had lower heterogeneities. These results may suggest that hyperuricemic therapy ameliorates $\beta$ cell function. In vitro studies showed that hyperuricemia may contribute to abnormal glucose metabolism by causing oxidative damage and function inhibition of pancreatic $\beta$ cells $^{5}$. An animal study suggested that hyperuricemia even could cause pancreatic $\beta$-cell death and dysfunction through nuclear factor-KB (NF-kB) signaling pathway, and the deleterious effects can be attenuated by allopurinol ${ }^{4}$. It was also demonstrated that allopurinol reduced the additional pro-inflammatory and anti-angiogenic responses to excess glucose through its inhibition of both IL-1 $\beta$ and ROS production by the trophoblast ${ }^{52}$. Furthermore, ULT with febuxostat for 12 weeks reduced the levels of FPG in the patients with hyperuricemia ${ }^{43}$. These results suggest that ULT could improve $\beta$-cell function. However, our results did not show that lowering of uric acid had any effect on HOMA- $\beta$. This may be due to inadequate trials for analyses. More studies are need for future analyses.

In addition, several studies also suggested that IR itself or compensatory hyperinsulinemia may lead to hyperuricemia and this seems to be a vicious circle. ${ }^{14,53,54}$ It is, therefore, important for hyperuricemic patients to lower uric acid levels regarding its potential risk for diabetes.

The findings of our study indicated that hypouricemic treatment may has a beneficial effect on BP, which could reduce cardiovascular risk. Recent cross-sectional and cohort studies have identified hyperuricemia is closely related with the development of hyperten$\operatorname{sion}^{5-59}$. Moreover, a randomized, double-blind, placebo-controlled, crossover trial demonstrated that treatment with allopurinol and probenecid both resulted in significant reductions of $\mathrm{BP}$, which means that uric acid was associated with increased BP that can be mitigated by ULT regardless by inhibition of uric acid production or acceleration of uric acid excretion ${ }^{59,60}$. Actually, agreeable with our study one meta-analysis also showed that ULT decreased both SBP and DBP ${ }^{61}$. The possible mechanisms that high uric acid-associated hypertension may be due to high levels of uric acid trigger arteriosclerosis since oxidative stress occurred during uric acid production, urate transporter disorders, and vascular disorders from hyperuricemia ${ }^{55}$. Weisman et $\mathrm{al}^{62}$ also found that ULT was associated with reduced mortality and cardiovascular outcomes, which may attributable to the alleviation in $\mathrm{BP}$.

Concerning serum lipids, our results suggested that uric acid lowering had no contribution to lipid metabolism. Several trials suggested that HUA is associated with TG and high-density lipoprotein-cholesterol (HDL-c), but not with TC or low-density lipoprotein-cholesterol (LDL-c) ${ }^{34,63,64}$. However, in our study, there is no statistically decrease neither in TG nor in TC levels with the reduction of uric acid. Interestingly, Kushiyama et al discovered that oral administration of allopurinol to ApoE knockout mice markedly ameliorated lipid accumulation and calcification in vivo, but serum lipid levels were not signifcantly altered by allopurinol65. Future physiological and prospective studies are needed for the causal links and underlying mechanisms between ULT with glucose and lipid metabolism.

\section{Strengths and limitation}

Previous studies showed that hyperuricemia is associated with insulin resistance and increases risk for diabetes $^{6,33}$. Our meta-analysis is the first one to demonstrate that uric acid lowering improved insulin resistance. Secondly, the study involved with 7 eligible trials with 503 participants and 4 of them had high or medium quality, and we did detailed analysis of the limitation of each trial. No evidence of substantial publication bias in all of these trials was founded. Finally, stratified analyses provided further evidence for our conclusion as we state above.

There are several limitations for the current study. Firstly, our study did not show that hypouricemic treatment improved $\beta$ cell functions although insulin resistance did be ameliorated. The main reason for this is due to limited number of trials containing the data for evaluation of $\beta$ cell functions. Secondly, in our study the publications included for meta-analysis were limited by the search strategy which aims at FPG, FINS and insulin resistance, but not at serum lipid or BP, which may lead to the in-complete evaluation of data for the cardiovascular risk factors. This may partially explain several re- 
sults of these outcomes in our study are different from others ${ }^{27,30,63}$. Thirdly, the heterogeneity was observed in the present study. This may be due to following reasons: (1) most of these study didn't aim to evaluate the effect of insulin sensitivity or $\beta$-cell function, thus generated some confounding factors, such as different treatment on diabetes; (2) different intervention methods in studies , such as different kinds or dosage of uric acid-lowering agents ; (3) sample size was not large enough.

\section{Conclusion}

Our meta-analysis of RCTs provided the evidence that uric acid-lowering in hyperuricemia patients significantly improved insulin sensitivity and lowered BP. Furthermore, the observation that ULT decreased fasting insulin levels in combination with improvement of insulin resistance may suggest that uric acid-lowering agents can ameliorate $\beta$-cell function. This may provide a new regimen for diabetes prevention and treatment, especially for diabetic patients with asymptomatic hyperuricemia.

\section{Abbreviations}

BP: blood pressure; CBM: Chinese Biomedical Literature Database; CI: confidence interval; DBP: diastolic blood pressures; FPG: fasting plasma glucose; FINS: fasting insulin; HDL-c: high-density lipoprotein-cholesterol; HOMA- $\beta$ : homeostasis model assessment of $\beta$-cell function; HOMA-IR: homeostasis model assessment of insulin resistance; HUA: high uric acid; IR: insulin resistance; IRS-1: antioxidant $\mathrm{N}$-acetylcysteine blocked HUA-induced activation of insulin receptor substrate 1; PRISMA: Preferred Reporting Items for Systematic Reviews and Meta-Analyses; RCTs: randomized controlled trials; SBP: systolic blood pressures; ROS: reactive oxygen species; SUA: serum uric acid; T2DM: type 2 diabetes mellitus; TC: total cholesterol; TG, triglyceride; ULT: urate lowering therapy; WMD: weighted mean differences.

\section{Ethics approval and consent to participate}

Not applicable.

\section{Consent for publication}

Not applicable.

\section{Availability of data and materials}

The datasets used and/or analysed during the current study are available from the corresponding author on reasonable request.

\section{Conflicts of interest}

The authors declare no conflicts of interest.

\section{Findings}

This study received no specific funding for this work.

\section{Author's contribution}

Tao Wang designed the study, interpreted data critically revised the manuscript and supervised the study. Qunchuan Zong originated the study, acquired data, analyzed statistics, interpreted data, drafted the manuscript, and critically revised the manuscript. Qunchuan Zong and Guanyi Ma analyzed statistics, interpreted data, and critically revised the manuscript. Qunchuan Zong and Tao Wang interpreted data and critically revised the manuscript for important intellectual content.

\section{Acknowledgments}

None.

\section{References}

1. Yamaoka-Tojo M, Tojo T, Takahira N, et al. Elevated circulating levels of an incretin hormone, glucagon-like peptide-1, are associated with metabolic components in high-risk patients with cardiovascular disease. Cardiovasc Diabetol. 2010;9: 1-9 PubMed.

2. Johnson RJ, Lanaspa MA, Gaucher EA. Uric Acid: A Danger Signal From the RNA World That May Have a Role in the Epidemic of Obesity, Metabolic Syndrome, and Cardiorenal Disease: Evolutionary Considerations. Semin Nephrol. 2011;31: 394-399 PubMed.

3. Zhu Y, Hu Y, Huang T, et al. High uric acid directly inhibits insulin signalling and induces insulin resistance. Biochem Biophys Res Commun. 2014;447: 707-714.

4. Jia L, Xing J, Ding Y, et al. Hyperuricemia Causes Pancreatic $\beta$-Cell Death and Dysfunction through NFxB Signaling Pathway. PLoS One. 2013;8.

5. Zhang Y, Yamamoto T, Hisatome I, et al. Uric acid induces oxidative stress and growth inhibition by activating adenosine monophosphate-activated protein kinase and extracellular signal-regulated kinase signal pathways in pancreatic $\beta$ cells. Mol Cell Endocrinol. 2013;375: 89-96 PubMed.

6. Wan X, Xu C, Lin Y, et al. Uric acid regulates hepatic steatosis and insulin resistance through the NLRP3 inflammasome-dependent mechanism. J Hepatol. 2016;64: 925-932 PubMed.

7. Adnan E, Rahman IA, Faridin HP. Relationship between insulin resistance, metabolic syndrome components and serum uric acid. Diabetes Metab Syndr Clin Res Rev. 2019;13: 2158-2162. 
8. Mortada I. Hyperuricemia, Type 2 Diabetes Mellitus, and Hypertension: an Emerging Association. Curr Hypertens Rep. 2017;19(9):69.

9. Kodama S, Saito K, Yachi Y, et al. Association between serum uric acid and development of type 2 diabetes. Diabetes Care. 2009;32: 1737-1742 PubMed.

10. Liu J, Tao L, Zhao Z, et al. Two-year changes in hyperuricemia and risk of diabetes: A five-year prospective cohort study. J Diabetes Res. 2018;2018.

11. Lv Q, Meng XF, He FF, et al. High Serum Uric Acid and Increased Risk of Type 2 Diabetes: A Systemic Review and Meta-Analysis of Prospective Cohort Studies. PLoS One. 2013;8: 1-7 PubMed.

12. Varadaiah YGC, Sivanesan S, Nayak SB, et al. Purine metabolites can indicate diabetes progression. Arch Physiol Biochem. 2019;0: 1-5.

13. Choi BG, Kim DJ, Baek MJ, et al. Hyperuricaemia and development of type 2 diabetes mellitus in Asian population. Clin Exp Pharmacol Physiol. 2018;45(6):499506.

14. Nakamura K, Sakurai M, Miura K, et al. HOMA-IR and the risk of hyperuricemia: A prospective study in non-diabetic Japanese men. Diabetes Res Clin Pract. 2014;106: 154-160.

15. Wu Y, He H, Yu K, et al. The association between serum uric acid levels and insulin resistance and secretion in prediabetes mellitus: A cross-sectional study. Ann Clin Lab Sci. 2019;49: 218-223 PubMed.

16. Testa A, Prudente S, Leonardis D, et al. A genetic marker of hyperuricemia predicts cardiovascular events in a meta-analysis of three cohort studies in high risk patients. Nutr Metab Cardiovasc Dis. 2015;25(12):10871094.

17. Woldeamlak B, Yirdaw K, Biadgo B. Hyperuricemia and its association with cardiovascular disease risk factors in type two diabetes mellitus patients at the University of Gondar Hospital, Northwest Ethiopia. Electron J Int Fed Clin Chem Lab Med. 2019;30: 325-339.

18. Kim SY, Guevara JP, Kim KM, Choi HK, Heitjan DF, Albert DA. Hyperuricemia and risk of stroke: a systematic review and meta-analysis. Arthritis Rheum. 2009;61(7):885-892.

19. $\mathrm{Xu} \mathrm{Y,} \mathrm{Zhu} \mathrm{J,} \mathrm{Gao} \mathrm{L,} \mathrm{et} \mathrm{al.} \mathrm{Hyperuricemia} \mathrm{as} \mathrm{an}$ Independent Predictor of Vascular Complications and Mortality in Type 2 Diabetes Patients: A Meta-Analysis. PLoS One. 2013;8.

20. Higgins JPT, Green S. Cochrane Handbook. Version 5.1.0. Http://HandbookCochraneOrg/. 2011.

21. Higgins JPT. Commentary: Heterogeneity in meta-analysis should be expected and appropriately quantified. Int J Epidemiol. 2008;37: 1158-1160 PubMed.
22. Higgins JP, Thompson SG, Deeks JJ AD. Measuring inconsistency in meta-analyses. BMJ. 2003;327: 557-660 PubMed.

23. DerSimonian R, Laird N. Meta-analysis in clinical trials revisited. Contemp Clin Trials. 2015;45: 139-145 PubMed.

24. Begg CB, Mazumdar M. Operating Characteristics of a Rank Correlation Test for Publication Bias. Biometrics. 1994;50: 1088.

25. Egger M, Smith GD, Schneider M, et al. Bias in meta-analysis detected by a simple, graphical test. BMJ. 1997;315: 629-34 PubMed.

26. Szwejkowski BR, Gandy SJ, Rekhraj S, et al. Allopurinol reduces left ventricular mass in patients with type 2 diabetes and left ventricular hypertrophy. J Am Coll Cardiol. 2013;62: 2284-2293 PubMed.

27. Perez-Pozo SE, Schold J, Nakagawa T, et al. Excessive fructose intake induces the features of metabolic syndrome in healthy adult men: Role of uric acid in the hypertensive response. Int J Obes. 2010;34: 454-461 PubMed.

28. Takir M, Kostek O, Ozkok A, et al. Lowering uric acid with allopurinol improves insulin resistance and systemic inflammation in asymptomatic hyperuricemia. J Investig Med. 2015;63: 924-929 PubMed.

29. Liu P, Wang H, Zhang F, et al. The effects of allopurinol on the carotid intima-media thickness in patients with type 2 diabetes and asymptomatic hyperuricemia: A three-year randomized parallel-controlled study. Intern Med. 2015;54: 2129-2137 PubMed.

30. Ding L, He W, Ji X. Effect of reducing serum uric acids on insulin resistance and islet $\beta$ cell functions in patients with hyperuricemia. Chin J Diabetes, 2012(07): 496-498 PubMed.

31. Le Z, The effect of controlling of hyperuricemia on HOMA-IR and glucose metabolism. Contemporary Medicine, 2013(22): 36-37.

32. Ogino K, Kato M, Furuse Y, et al. Uric acid-lowering treatment with benzbromarone in patients with heart failure a double-blind placebo-controlled crossover preliminary study. Circ Hear Fail. 2010;3: 73-81 PubMed.

33. Wu Y, He H, Yu K, et al. The association between serum uric acid levels and insulin resistance and secretion in prediabetes mellitus: A cross-sectional study. Ann Clin Lab Sci. 2019;49: 218-223 PubMed.

34. Li Q, Yang Z, Lu B, et al. Serum uric acid level and its association with metabolic syndrome and carotid atherosclerosis in patients with type 2 diabetes. Cardiovasc Diabetol. 2011;10: 1-7 PubMed.

35. Johnson RJ, Nakagawa T, Sanchez-Lozada LG, 
et al. Sugar, uric acid, and the etiology of diabetes and obesity. Diabetes 2013;62:3307-3315 PubMed

36. Hu QH, Wang C, Li JM, et al. Allopurinol, rutin, and quercetin attenuate hyperuricemia and renal dysfunction in rats induced by fructose intake: Renal organic ion transporter involvement. Am J Physiol - Ren Physiol. 2009;297: 1080-1091 PubMed.

37. Li X, Meng X, Gao X, et al. Elevated serum xanthine oxidase activity is associated with the development of type 2 diabetes: A prospective cohort study. Diabetes Care 2018;41(4):884-90. PubMed

38. Yisireyili M, Hayashi M, Wu H, et al. Xanthine oxidase inhibition by febuxostat attenuates stress-induced hyperuricemia, glucose dysmetabolism, and prothrombotic state in mice. Sci Rep. 2017;7(1):1266 PubMed.

39. Meng J, Li Y, Yuan X, et al. Effects of febuxostat on insulin resistance and expression of high-sensitivity C-reactive protein in patients with primary gout. Rheumatol Int. 2017;37: 299-303 PubMed.

40. Tatar E, Demirci MS, Kircelli F, et al. Association of insulin resistance with arterial stiffness in nondiabetic peritoneal dialysis patients. Int Urol Nephrol. 2012;44: 255-262 PubMed.

41. Manco M, Nolfe G, Calvani M, et al. Menopause, insulin resistance, and risk factors for cardiovascular disease. Menopause. 2006;13: 809-817 PubMed.

42. Chiou WK, Wang MH, Huang DH, et al. The relationship between serum uric acid level and metabolic syndrome: Differences by sex and age in Taiwanese. $J$ Epidemiol. 2010;20: 219-224 PubMed.

43. Barbieri L, Verdoia M, Schaffer A, et al. Uric acid levels and the risk of Contrast Induced Nephropathy inpatients undergoing coronary angiography or PCI. Nutr Metab Cardiovasc Dis. 2015;25: 181-186. doi:10.1016/j. numecd.2014.08.008

44. Gu Y, Han B, Wang L, et al. Low Serum Levels of Uric Acid are Associated with Development of Poststroke Depression. Med (United States). 2015;94: e1897. 45. Guo M, Niu JY, Li SR, et al. Gender differences in the association between hyperuricemia and diabetic kidney disease in community elderly patients. J Diabetes Complications. 2015;29: 1042-1049.

46. Lohsoonthorn V, Dhanamun B, Williams MA. Prevalence of Hyperuricemia and its Relationship with Metabolic Syndrome in Thai Adults Receiving Annual Health Exams. Arch Med Res. 2006;37: 883-889 PubMed.

47. Hatoum H, Khanna D, Lin SJ, et al. Achieving serum urate goal: A comparative effectiveness study between allopurinol and febuxostat. Postgrad Med. 2014;126: 6575 PubMed.

48. Hare JM, Mangal B, Brown J, et al. Impact of Oxypurinol in Patients With Symptomatic Heart Failure. Results of the OPT-CHF Study. I Am Coll Cardiol. 2008;51: 2301-2309 PubMed.

49. Wu AH, Ghali JK, Neuberg GW, et al. Uric acid level and allopurinol use as risk markers of mortality and morbidity in systolic heart failure. Am Heart J. 2010;160: 928-933 PubMed.

50. Anter AH, Abdel-Latif RM. The safety and efficacy of fluorouracil, leucovorin, oxaliplatin, and docetaxel (FLOT) combination in the front-line treatment for patients with advanced gastric or gastroesophageal adenocarcinoma: Phase II trial. Med Oncol. 2013;30: 1-6 PubMed.

51. Joosten LAB, Crişan TO, Bjornstad P, et al. Asymptomatic hyperuricaemia: a silent activator of the innate immune system. Nat Rev Rheumatol. 2020;16: 75-86 PubMed .

52. Negi M, Mulla MJ, Han CS, et al. Allopurinol inhibits excess glucose-induced trophoblast IL-1 $\beta$ and ROS production. Reproduction. 2020;159: 73-80 PubMed .

53. Iba A, Kohjimoto Y, Mori T, et al. Insulin resistance increases the risk of urinary stone formation in a rat model of metabolic syndrome. BJU Int. 2010;106: 1550-1554 PubMed.

54. Li C, Hsieh MC, Chang SJ. Metabolic syndrome, diabetes, and hyperuricemia. Curr Opin Rheumatol. 2013; 25: 210-216 PubMed.

55. Ito $\mathrm{H}$, Abe M, Mifune M, et al. Hyperuricemia Is Independently Associated with Coronary Heart Disease and Renal Dysfunction in Patients with Type 2 Diabetes Mellitus. PLoS One. 2011;6: 1-7 PubMed.

56. Kuwabara M. Hyperuricemia, Cardiovascular Disease, and Hypertension. Pulse. 2016;3: 242-252 PubMed.

57. Xia X, Zhao C, Peng FF, et al. Serum uric acid predicts cardiovascular mortality in male peritoneal dialysis patients with diabetes. Nutr Metab Cardiovasc Dis. 2016;26: 20-26.

58. Feig DI, Madero M, Jalal DI, et al. Uric acid and the origins of hypertension. J Pediatr. 2013;162: 896-902 PubMed.

59. Soletsky B, Feig DI. Uric acid reduction rectifies prehypertension in obese adolescents. Hypertension. 2012;60: 1148-1156 PubMed. doi:10.1161/HYPERTENSIONAHA.112.196980

60. Feig DI, Soletsky B, Johnson RJ. Effect of allop- 
urinol on blood pressure of adolescents with newly diagnosed essential hypertension: A randomized trial. JAMA - J Am Med Assoc. 2008;300: 924-932 PubMed. 61. Agarwal V, Hans N, Messerli FH. Effect of Allopurinol on Blood Pressure: A Systematic Review and Meta-Analysis. J Clin Hypertens. 2013;15: 435-442 PubMed.

62. Weisman A, Tomlinson GA, Lipscombe LL, et al. Association between allopurinol and cardiovascular outcomes and all-cause mortality in diabetes: A retrospective, population-based cohort study. Diabetes, Obes Metab. 2019;21: 1322-1329 PubMed.
63. Kawamoto R, Tomita H, Oka Y, et al. Relationship between serum uric acid concentration, metabolic syndrome and carotid atherosclerosis. Intern Med. 2006;45: 605-614 PubMed.

64. Chiou WK, Huang DH, Wang MH, et al. Significance and association of serum uric acid (UA) levels with components of metabolic syndrome (MS) in the elderly. Arch Gerontol Geriatr. 2012;55: 724-728.

65. Kushiyama A, Okubo H, Sakoda H, et al. Xanthine oxidoreductase is involved in macrophage foam cell formation and atherosclerosis development. Arterioscler Thromb Vasc Biol. 2012;32: 291-298. 